\title{
The Drexel Defusion Scale: Reliability and Validity of the Turkish Version*
}

Gökçen AYDIN**

\author{
Oya YERIN GÜNERI***
}

\begin{abstract}
Cognitive defusion is one of the components of psychological flexibility in Acceptance and Commitment Therapy. The purpose of this study was to translate the Drexel Defusion Scale (DDS) into Turkish and test its psychometric properties. This two-phase study was carried out with two groups of participants. The first phase, Exploratory Factor Analysis (EFA) included 370 university students (218 female, 152 male). The second phase, Confirmatory Factor Analysis (CFA) and divergent validity consisted of 715 university students (351 female, 364 male). The DDS measures the ability to achieve a distance from inner experiences like thoughts and feelings, and it consists of 10 scenarios on a six-point Likert-scale ranging from 0 (Not at all) to 5 (Very much). The DDS has a unidimentional factor structure. The CFA results confirmed the one-factor structure of Turkish DDS (T-DDS). The T-DDS also yielded satisfactory $(\alpha>.80)$ internal consistency and test-retest reliability $(\alpha=$ .81). Thus, findings revealed satisfactory reliability and validity evidence for the TDDS.
\end{abstract}

Keywords: Cognitive defusion, adaptation, reliability, validity.

\footnotetext{
${ }^{*}$ Paper presented at 27th International Conference on Educational Sciences (ICES-UEBK), p. 3769-3770. Antalya, Turkey

The ethics committee approval for this study was obtained from the Ethics Committee of the Rectorate of Middle East Technical University, dated 13/04/2015 and numbered 28620816/177-361.

** Orcid ID: https://orcid.org/0000-0003-0781-7817, Assist. Prof. Dr., Hasan Kalyoncu University, Turkey, gokcenaydn@gmail.com

${ }_{* * *}$ Orcid ID: https://orcid.org/0000-0002-0565-4025, Prof. Dr., Middle East Technical University, Turkey, guneri@metu.edu.tr
}

Aydın, G., \& Yerin Güneri, O. (2021). The drexel defusion scale: reliability and validity of the Turkish version, Sakarya University Journal of Education, 11(1), 67-82. doi: https://doi.org/10.19126/suje.770582 


\section{INTRODUCTION}

With its complex psychological processes, our mind lies at the core of our suffering. Thus, Acceptance and Commitment Therapy (ACT) gives importance to acceptance, compassion, forgiveness, mindfulness, and taking values-based actions to live a rich and meaningful life and ease human suffering (Harris, 2006). The ACT recognizes six strongly related core processes that contribute to psychological inflexibility: experiential avoidance; cognitive fusion; emphasis on past and future; attachment to conceptualized self; lack of values; inaction, impulsivity and, avoidant persistence. Among these, cognitive fusion refers to the inclination of human beings to be immersed in the content of their thoughts or to take them literally. This tendency causes people to act according to their thoughts about an event rather than respond to the event itself (Luoma, Hayes, \& Walser, 2007). In contrast to cognitive fusion, cognitive defusion helps individuals to distance themselves from their thoughts by accepting those thoughts as "only thoughts" and to live in the present moment by following their values (Hayes, Luoma, Bond, Masuda, \& Lillis, 2006). The cognitive defusion includes changing the relationship with the thoughts instead of being overwhelmed with them. The ACT stresses the fact that stopping thinking or releasing an idea is impossible; yet, the function of context can be switched even if relations between events cannot be changed (Hayes et al., 2006). Cognitive defusion supports a healthy way of human functioning by thinking oneself outside the problem from a distance (Hayes \& Lillis, 2012).

A wide range of research has been conducted to investigate the relationship between cognitive defusion, physical or psychological health among clinical and non-clinical samples. For example, cognitive defusion was found to be negatively related with negative thoughts (Deacon, Fawzy, Lickel, \& Wolitzky-Taylor, 2011; Masuda et al., 2010; Larsson, Hooper, Osborne, Bennett, \& McHugh, 2016), tinnitus (Hesser, Westin, Hayes, \& Andersson, 2009), psychological distress and low self-esteem (Hinton \& Gaynor, 2010), negative self-statements (Healy et al., 2008), learned helplessness (Hooper \& McHugh, 2013), negative body image (Mandavia et al., 2015), and eating disorders (Moffitt, Brinkworth, Noakes, \& Mohr, 2012) and to be positively with social and mental health functioning (McCracken, Barker, \& Chilcot, 2014), and quality of life (Forman et al., 2012).

Experimental studies have also explored the effects of cognitive defusion training on emotional distress. For example, Masuda et al. (2010) showed that cognitive defusion training decreased emotional discomfort, negative thoughts and symptoms of depression in university students. In another study by Pilecki and Mckay (2012) that classified undergraduate students into three groups (cognitive defusion, thought suppression, control), cognitive defusion was found to reduce emotional distress, even after controlling for individual differences in negative affect. The authors concluded that while cognitive defusion did not stop negative emotions, it did make them easier to handle. Cognitive defusion and mindfulness strategies were also found effective in alleviating depression and anxiety in older adults (Roberts \& Sedley, 2016). 
Several measures exist for assessing cognitive defusion. First, The Toronto Mindfulness Scale-Decentering Subscale (TMS-D) measures state of awareness, distance from thoughts and internal experience (Lau et al., 2006). The scale has seven items on a fivepoint scale. Second, Gillander et al. (2014) developed the Cognitive Fusion Questionnaire (CFQ) to measure fusion by cognition, controlling cognitions and deep analysis of thoughts. The scale has 42 items on a seven-point scale. They tested the reliability and validity with a sample of more than 1,800 clinical and non-clinical participants. Also, The Believability of Anxious Feelings and Thoughts Questionnaire (BAFT) was developed by Herzberg et al. (2012) to measure cognitive fusion especially for anxiety disorders. The scale has 16 items rated on seven-point Likert scale. BAFT was tested on 935 undergraduate students and found to be a reliable and valid instrument for use in both clinical and non-clinical samples. Finally, The Drexel Defusion Scale (DDS), which also assesses the ability to achieve a distance from inner experiences like thoughts and feelings (Forman et al., 2012), has 10 scenarios on a six-point Likert type scale. DDS was found to be reliable and valid in a sample of university students, and the scale was later adapted to Portuguese (Carvalho, Castilho, Pinto-Gouveia, 2013). DDS is slightly different from other measures with having scenarios rather than items. The scenarious make items more understandable for the subjects who are not familiar with the concept. Forman et al. (2012) underlined the need for future studies that would undertake confirmatory factor analysis of the DDS with larger sample sizes as well as the importance of adapting the DDS into other languages.

Considering the extensive use of Acceptance and Commitment Therapy (ACT) worldwide, measuring the core concepts of psychological flexibility is recognized in different countries. However, in previous ACT studies, cognitive defusion has generally been measured within psychological flexibility, decentering, or acceptance. Thus, the research in which cognitive defusion is examined as a separate variable of psychological flexibility is limited. The use of cognitive defusion with clinical and non-clinical samples to open an open stance towards one's internal experiences requires reliable and valid instruments. In recent years, there has been an increase in ACT research in Turkey. Various ACT measures have also been either developed or adapted to Turkish by researchers. Although the Toronto Mindfulness Scale-Decentering Subscale has translated into Turkish (Hisli Şahin \& Yeniçeri, 2015), it does not directly measure cognitive defusion. In fact, there is no scale in Turkish for measuring cognitive defusion. Thus, taking into account the suggestions in the literature and given the importance of studying the construct and related variables in different cultures, this study aimed to adapt the DDS into Turkish and test its psychometric properties among university students. 


\section{METHOD}

\section{The Study Group}

A total of 1,085 students attending the Department of Basic English (DBE) at a state university in Turkey participated in the study. English is the medium of instruction at this university, and the DBE provides one year of intensive English-language instruction to almost all new coming students who have been conditionally accepted into the undergraduate program provided that they achieve proficiency in English, with proficiency determined based on an end-of-year examination. In the present study, data were collected by convenient sampling method via the online survey system of university. In the first phase of the study, the Exploratory Factor Analysis (EFA) included 370 students (218 female, 152 male); and the second phase, Confirmatory Factor Analysis (CFA), was conducted with 715 students (351 female, 364 male). The mean age of participants changed between 17-27 years $(M=18.57, S D=1.02)$. Table 1 shows demographic information of participants included in the study.

Table 1

Demographic Information of the Participants

\begin{tabular}{clll}
\hline \multicolumn{1}{l}{ Variables } & $f$ & $\%$ \\
\hline Gender & & & \\
\cline { 2 - 3 } & Female & 569 & 52.44 \\
Male & 516 & 47.56 \\
Language Level & & \\
Beginner & 246 & 22.67 \\
Elementary & 343 & 31.61 \\
Pre-intermediate & 96 & 8.85 \\
Intermediate & 307 & 28.29 \\
Upper-Intermediate & 93 & 8.58 \\
\hline
\end{tabular}

\section{Data Collection Tools}

The data were collected using demographic information form (age and gender), the Turkish version of the Drexel Defusion Scale (T-DDS), and Acceptance and Action Questionnaire-II (AAQ-II).

Drexel Defusion Scale (DDS): The DDS (Forman et al., 2012) measures the ability to achieve a distance from inner experiences like thoughts and feelings with 10 items, rated on a six-point Likert-scale ranging from 0 (Not at all) to 5 (Very much). The DDS gives a total score in which a high score indicates a good state of psychological distance from thoughts/feelings, and a low score indicates an inability to defuse from 
thoughts/feelings. The scale does not have reverse items. The original DDS was tested across two different samples. In the first phase of the study, the sample was composed of 235 non-clinical undergraduate students ranging in age from 18-42 years, whereas in the second phase of the study, the sample consisted of 144 students ranging in age from 18 to 53 who were receiving support from the university counseling center $(M=26.0)$.

The DDS was found to have good internal consistency (.80 - .83) (Forman et al., 2012), and a high convergent and divergent validity as measured by the Philadelphia Mindfulness Scale (Cardaciotto, Herbert, Forman, Moitra, \& Farrow, 2008); the Kentucky Inventory of Mindfulness Skills (Baer, Smith, \& Allen, 2004); the Acceptance and Action Questionnaire-2 (AAQ-2; Bond et al., 2011); the Experiences Questionnaire (Fresco et al., 2007); the Quality of Life Inventory (Frisch, 1994); the Brief Symptom Inventory (Derogatis \& Melisaratos, 1983) (Forman et al., 2012). EFA was conducted using Principal Component Analysis as well as Promax Rotation and indicated a unidimensional factor structure; however, CFA was not conducted in the original development study (Forman et al., 2012).

Acceptance and Action Questionnaire-II (AAQ-II) (Bond et al., 2011): Hayes et al. (2004) developed a 16-item Acceptance and Action Questionnaire (AAQ) to measure psychological inflexibility. Later, Bond et al. (2011) revised the scale and named it as AAQ-II. The AAQ-II has seven items rated on a 7-point Likert scale (1: Never true to 7: Always true). Higher scores show a high level of psychological inflexibility. AAQ-II has a unidimensional factor structure. The Cronbach's alpha and test-retest reliability were reported as .84 and .81, respectively (Bond et al., 2011). The AAQ-II was adapted into Turkish by Meunier et al. (2014). Internal consistency was calculated as .88 and testretest reliability as .78 in two-month interval for the Turkish version of AAQ-II (Meunier et al., 2014).

\section{Process}

The spring semester of the 2015-2016 academic year was the time of the data collection following approval by the Human Subjects Ethics Committee from Middle East Technical University, dated 13/04/2015 and numbered 28620816/177-361. Students were initially informed about the study and invited to participate through the university web site. Interested students were able to participate in the first phase by clicking on an online survey link and completing it. The second phase of the study included the administration of the T-DDS in a paper-pencil format by classroom instructors, with the principal investigator informing instructors one week before data collection about the purpose of the study and how to administer the measure.

\section{Data Analysis}

Data were analysed using the software programs SPSS 24 and LISREL 8.80. Skewness and kurtosis values showed no violation regarding assumptions of univariate normality. However, the results of Mardia's test $(p<.05)$ indicated the violation of multivariate normality assumption. Consequently, to reduce the effects of non-normality on the CFA 
model, asymptotic covariances were calculated by performing a Satorra-Bentler correction in LISREL. Univariate outliers were found by $\mathrm{z}$ scores and multivariate outliers by Mahalanobis distance (Kline, 2011). The assumption of linearity was visually checked through scatter plots, and no violation was gathered. CFA was utilized to confirm the factor structure of the T-DDS. The Chi-square/df ratio, comparative fit index (CFI), goodness of fit index (GFI), and root mean square error of approximation (RMSEA) was calculated to test the fitness of the model, with the following reference points for goodness of fit: Chi-square/df ratio $\leq 5$; CFI and GFI $\geq .90$; RMSEA $\leq .08$ (Hu \& Bentler, 1999; Kline, 2011; Schumacker \& Lomax, 2010). In order to assess the internal consistency of the T-DDS, Cronbach's alpha coefficient was calculated. Finally, test-retest reliability was used to measure the stability of the T-DDS scores after a two-week interval.

\section{FINDINGS}

\section{Turkish Adaptation of the Drexel Defusion Scale (T-DDS)}

After receiving written permission from the author of the Drexel Defusion Scale (E. M. Forman) to translate and adapt the measure into Turkish, independent translation from English to Turkish was carried out by five experts, namely three advanced Ph.D. students from the field of counseling who were also proficient in English, and two Englishlanguage teachers, each with seven years of teaching experience in the field. The different translations were examined by the researchers, who selected the best fitting translation for each item, and the selections were checked for clarity and structure by a native-Turkish-speaking English-language expert with a B.A. in English-language teaching and a Ph.D. in Curriculum and Instruction. Given the crucial need to accurately reflect the scenarios presented in the scale, the language experts consulted with the researchers to confirm the clarity of the translations, and all translated items were approved by the language expert.

The clarity of the translated items was confirmed by conducting cognitive interviews with five undergraduate students who were instructed to read each of the translated items and evaluate them for clarity. During the interviews, which lasted approximately 20 minutes each, the students stated that the translated items were in general clear and understandable with one exception, namely that they had difficulty in understanding the meaning of the word 'defusion' ('ayrışma'). For this reason, in the final version of the scale, a synonymous phrase, 'put a distance' ('araya mesafe koyma'), was added in parenthesis after the word 'ayrışma' at the end of each item.

\section{Construct Validity Evidence}

\section{Exploratory Factor Analysis}

EFA was conducted with the first group of participants that was composed of 370 university students. As in the original DDS study (Forman et al., 2012), Principal 
Component Analysis with Promax Rotation was used to examine the factor structure of the T-DDS. KMO (.78) and Barlett's Test of Sphericity (.00) values indicated the suitability of the data for factor analysis. Similar to the original DDS scale (Forman et al., 2012), Eigenvalues for the T-DDS indicated a two-factor solution (2.93 and 1.26, respectively), and Promax Rotation found that these two factors accounted for $41.99 \%$ of variance (accounting for $29.37 \%$ and $12.62 \%$ with the first and second factor respectively). On the other hand, the scree plot suggested a one-factor solution (See Figure 1), which was also quite similar to the original DDS study. Table 2 presents the factor loadings.

Table 2

Factor Loadings and Communalities of T-DDS

\begin{tabular}{cc}
\hline Item Number & Factor 1 \\
\hline DDS6 & .76 \\
DDS5 & .71 \\
DDS7 & .69 \\
DDS8 & .68 \\
DDS4 & .65 \\
DDS10 & .43 \\
DDS9 & .41 \\
DDS3 & .38 \\
DDS2 & .37 \\
DDS1 & .37 \\
\hline
\end{tabular}




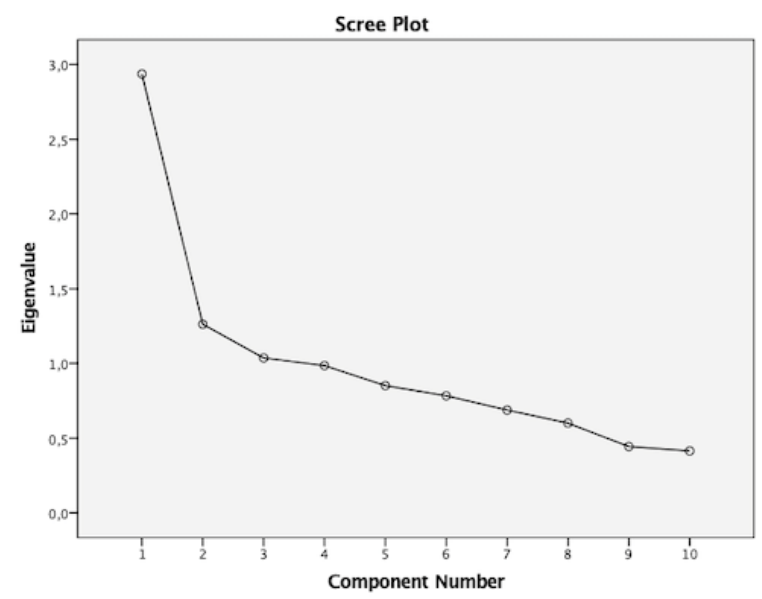

Figure 1. Scree plot of T-DDS

\section{Confirmatory Factor Analysis of DDS}

In the second phase, the purpose was to confirm the one-factor structure of the T-DDS; therefore, CFA was conducted with the second group of participants that was composed of 715 students. Prior to the main analysis, even though basic assumptions of univariate normality indicated no violations, the results of Mardia's test revealed a violation of multivariate normality $(p<.05)$. Consequently, Satorra-Bentler chi-square was calculated rather than normal chi-square analysis.

Similar to the EFA findings, CFA results confirmed the unidimensional structure of the TDDS in a sample of university students [Satorra-Bentler $\chi^{2}(33)=53.49, p=.00 ; \chi^{2} / d f$ ratio $=1.62 ; G F I=.97, C F I=.98, R M S E A=.04]$, with slight modifications between Items 78 and Items 9-10 (See Figure 2). Results indicated a perfect goodness of fit: Chisquare/df ratio $\leq 5$; GFI and CFI $\geq .90$; RMSEA $\leq .08$ (Hu \& Bentler, 1999; Kline, 2011; Schumacker \& Lomax, 2010). 


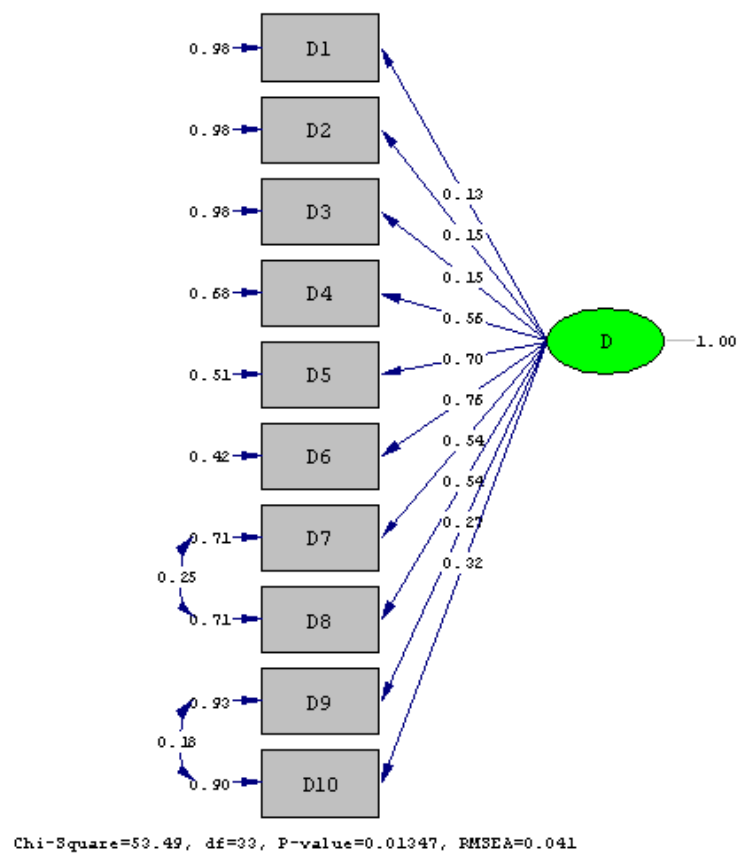

Figure 2. The Coefficients in Standardized Values for T-DDS

With respect to further analyses, standardized factor loadings varied between .12 and .73, and all items had significant t-values. The explained variance for each item ranged between 2\% - 58\% ( $\mathrm{R}^{2}$ ) (see Table 3). Although the standardized factor loadings of the first three items of the scale were relatively low, they were retained in the scale for the confirmation study as a result of significant $t$-values. The low level of variance explained by Item 1, Item 2, and Item 3 was attributed to the low levels of standardized loadings.

Table 3

Parameter Estimates of T-DDS

\begin{tabular}{llll}
\hline Item & $\begin{array}{l}\text { Standardized Factor } \\
\text { Loadings }\end{array}$ & $t$ & $R^{2}$ \\
\hline D1 & .12 & $2.03^{*}$ & .02 \\
D2 & .16 & $2.39^{*}$ & .02 \\
D3 & .16 & $2.56^{*}$ & .02 \\
D4 & .55 & $10.58^{*}$ & .32 \\
D5 & .67 & $15.19^{*}$ & .49 \\
D6 & .73 & $16.37^{*}$ & .58
\end{tabular}




\begin{tabular}{llll} 
D7 & .62 & $9.92^{*}$ & .29 \\
D8 & .61 & $10.16^{*}$ & .29 \\
D9 & .30 & $4.52^{*}$ & .07 \\
D10 & .31 & $5.68^{*}$ & .10 \\
\hline$p<.05$ & &
\end{tabular}

During the development of the original scale (Forman et al., 2012), the first EFA also yielded a two-factor solution, with Items 1,2 and 3 loading on one factor and demonstrating relatively low loadings compared to the other seven items, which loaded onto the second factor with strong loadings (Forman et al., 2012). However, further analysis supported a one-factor solution, and the authors concluded that the scale has a unidimensional structure. The findings were similar for the Portuguese version of the scale (Carvalho et al., 2013), for which the first three items also had low loadings. In the Portuguese version, the researchers tested the CFA by both omitting the first three items and making adjustments between items. When they correlated the errors, they concluded that the scale had good fit indexes and thus decided not to omit any items, and finally, the analysis was completed with a 10 -item scale. The authors did, however, recommend that further analytical studies be conducted, particularly with different samples (the Portuguese study was conducted with a sample derived from the general population, not a sample of university students), and with close attention paid to the loadings of the first three items (Carvalho et al., 2013).

In this study, EFA factor loadings were above .32, and CFA also yielded significant tvalues; therefore, the first three items (Forman et al., 2012, p. 64) (Item 1: "Feelings of anger. You become angry when someone takes your place in a long line. To what extent would you normally be able to defuse from feelings of anger?"; Item 2: "Cravings for food. You see your favorite food and have the urge to eat it. To what extent would you normally be able to defuse from cravings for food?"; and Item 3: "Physical pain. Imagine that you bang your knee on a table leg. To what extent would you normally be able to defuse from physical pain?") were not omitted from the scale during CFA. Ultimately, it was concluded that the standard estimates, t-values, and variance explained support a one-factor structure of the T-DDS for the current study sample.

\section{Divergent Validity}

The Turkish version of AAQ-II (Meunier et al., 2014) was used to assess the construct validity of T-DDS. The strength of the association between T-DDS and Turkish version of AAQ-II, a measure of psychological inflexibility, was examined. Kline (2011) suggested the criteria of correlation below .85 for divergent validity, and Swank and Mullen (2017) pointed out that negative correlation indicates different constructs. The Pearson's $r$ analyses revealed a significant and negative correlation between T-DDS and Turkish 
version of AAQ-II $(r=-.47, \mathrm{p}<.01)$. It could be concluded that T-DDS seems to measure a related but different construct compared to AAQ-II.

\section{Reliability Evidence}

The Cronbach's alpha coefficient calculated for internal consistency of the T-DDS indicated high consistency $(\alpha=.80)$. Test-retest reliability was measured by administering the scale to 52 (25 female and 27 male) university students in English Language Preparatory School and re-applying it to the same participants two weeks later. The results yielded test-retest reliability of .81 for the T-DDS.

\section{RESULTS, DISCUSSIONS AND SUGGESTIONS}

Cognitive defusion is one of the core concepts of Acceptance and Commitment Therapy. As the use of this approach becomes widespread, the adaptation of measures of its related constructs to other languages gains more importance. Thus, this study focused on adapting the DDS into Turkish and testing its psychometric properties in a sample of university students. The T-DDS, is the first scale available in Turkish for measuring cognitive defusion.

The Exploratory Factor Analysis (EFA) of the T-DDS, as well as the Confirmatory Factor Analysis (CFA), yielded satisfactory results. Although the standardized values were low for Items 1, 2 and 3 as in the original as well as the Portuguese versions of the scale (Carhalvo et al., 2013; Forman et al., 2012), due to acceptable loadings obtained in Exploratory Factor Analysis and the significant t-values, the items were retained in the scale. The one-factor solution of the T-DDS was confirmed, and a good internal consistency coefficient was recorded. In conclusion, results yielded satisfactory evidence for the reliability and validity of this unidimensional scale. Considering that thoughts about self, thoughts about the future, and anxious thoughts are common reasons for people in all cultures to seek psychological help, it was expected that the factor structure of the scale would be confirmed in another culture.

However, Briggs (2019) replicated and extended the psychometric properties of the DDS with a large sample $(n=887)$ and found a two-factor structure. The Principal Components Analysis, with Promax and Varimax rotation, was conducted. The results yielded a two-factor solution. While the first three items loaded on the first factor remaining were loaded on the second one. According to Briggs (2019), the first three items of the scale demanded the person to measure the defusion level in a specific situation. However, the remaining items were much more general and related to thoughts, not very specific. Briggs (2019) underlined the need for convergent and divergent validity of the DDS in diverse samples.

The context in which an item is presented is an important factor in determining whether or not it is understood in the correct way. When studying the adaptation of the DDS into other languages, it is important to remember that cognitive diffusion can be a difficult 
concept to understand (Gillander, 2014). In the present study, a definition of the concept of "defusion" was provided at the beginning of the DDS scale to ensure that the items on the scale were easily understood, and participants were asked to respond to imagined scenarios rather than to relate their actual experiences.

Adapting T-DDS is a contribution for further studies about Acceptance and Commitment Therapy in Turkey because, among six ACT concepts, there has been no measure developed or adapted into Turkish about cognitive defusion. The availability of T-DDS opens up several implications for research and practice. The T-DDS can be used in future studies to investigate cognitive defusion and related variables with university students. Researchers can also use this newly adapted measure for criterion validity in the adaptation of other scales testing cognitive defusion in Turkish. Reliability and validity studies could also be conducted with different samples, including adults in clinical as well as non-clinical settings. Practitioners in university counseling centers can also use T-DDS to assess the cognitive defusion level of their clients before or after their ACTbased individual or group counseling interventions.

The findings also need to be interpreted with caution due to some limitations of the current study. First, the T-DDS relies on self-reports of the participants. Moreover, all of the study participants were students in the English Language Preparatory School of a state university in Turkey, and the results cannot be generalized to students at different class levels and students in other universities and other populations in Turkey. Therefore, in the future, the Turkish version of the scale should be tested with university students in different classes and also in other universities and with participants from other age groups.

\section{References}

Baer, R. A., Smith, G. T., \& Allen, K. B. (2004). Assessment of mindfulness by self-report: The Kentucky Inventory of Mindfulness Skills. Assessment, 11(3), 191-206. doi: $10.1177 / 1073191104268029$

Bond, F. W., Hayes, S. C., Baer, R. A., Carpenter, K. M., Guenole, N., Orcutt, H. K., ... , \& Zettle, R. D. (2011). Preliminary psychometric properties of the Acceptance and Action Questionniare-II: A revised measure of psychological flexibility and experiential avoidance. Behavior Therapy, 42, 676-688. doi: 10.1016/j.beth.2011.03.007

Briggs, C. A. (2019). Measurement of defusion: Further validation of the Drexel Defusion Scale. (Unpublished Doctoral Dissertation), Western Michigan University, https://scholarworks.wmich.edu/dissertations/3535

Cardaciotto, L., Herbert, J. D., Forman, E. M., Moitra, E., \& Farrow, V. (2008). The assessment of present-moment awareness and acceptance: The Philadelphia Mindfulness Scale. Assessment, 15(2), 204-223. doi: 10.1177/1073191107311467

Carvalho, S., Castilho, P., \& Pinto-Gouveia, J. (2013). The Portuguese version of The Drexel Defusion Scale: A validation study in clinical and non-clinical samples. Poster presented 
at Acceptance and Commitment Therapy (ACT) Conference and Workshops, London, UK.

Deacon, B. J., Fawzy, T. I., Lickel, J. J., \& Wolitzky-Taylor, K. B. (2011). Cognitive defusion versus cognitive restructuring in the treatment of negative self-referential thoughts: An investigation of process and outcome. Journal of Cognitive Psychotherapy, 25(3), 218-232. doi:10.1891/0889-8391.25.3.218

Derogatis, L. R., \& Melisaratos, N. (1983). The brief symptom inventory: An introductory report. Psychological Medicine, 13(3), 595-605.

Forman, E. M., Herbert, J. D., Juarascio, A. S., Yeomans, P. D., Zebell, J. A., Goetter, E. M., \& Moitra, E. (2012). The Drexel Defusion Scale: A new measure of experiential distancing. Journal of Contextual Behavioral Science, 1(1-2), 55-65. doi: 10.1016/j.jcbs.2012.09.001

Fresco, D. M., Moore, M. T., van Dulmen, M. H., Segal, Z. V., Ma, S. H., Teasdale, J. D., \& Williams, J. M. G. (2007). Initial psychometric properties of the experiences questionnaire: Validation of a self-report measure of decentering. Behavior therapy, 38(3), 234-246. doi: $10.1016 /$ j.beth.2006.08.003

Frisch, M. B. (1994). Quality of life inventory (QOLI). Minneapolis, MN: National Computer Systems.

Gillanders, D.T., Bolderston, H., Bond, F.W., Dempster, M., Flaxman, P.E., Campbell, L., ... \& Masley, S. (2014). The development and initial validation of the Cognitive Fusion Questionnaire. Behavior Therapy, 45(1), 83-101. doi: 10.1016/j.beth.2013.09.001

Harris, R. (2006). Embracing your demons: An overview of acceptance and commitment therapy. Psychotherapy in Australia, 12(4). doi: 10.3316/informit.545561433272993

Hayes, S. C., \& Lillis, J. (2012). Acceptance and commitment therapy: Theories of psychotherapy series. Washington, DC: American Psychological Association.

Hayes, S. C., Luoma, J. B., Bond, F. W., Masuda, A., \& Lillis, J. (2006). Acceptance and commitment therapy: Model, processes and outcomes. Behaviour Research and Therapy, 44(1), 1-25. doi:10.1016/j.brat.2005.06.006

Hayes, S. C., Strosahl, K., \& Wilson, K. G. (1999). Acceptance and commitment therapy: An experiential approach to behavior change. New York: The Guilford Press.

Hayes, S. C., Strosahl, K., Wilson, K. G., Bissett, R. T., Pistorello, J., Toarmino, D., ... M.Mccurry, S. (2004). Measuring experiential avoidance: A preliminary test of a working model. The Psychological Record, 54(4), 553-578. doi:10.1017/CB09781107415324.004

Healy, H. A., Barnes-Holmes, Y., Barnes-Holmes, D., Keogh, C., Luciano, C., \& Wilson, K. (2008). An experimental test of a cognitive defusion exercise: Coping with negative and positive self-statements. The Psychological Record, 58(4), 623-640. doi: 10.1007/BF03395641

Hesser, H., Westin, V., Hayes, S. C., \& Andersson, G. (2009). Clients' in-session acceptance and cognitive defusion behaviors in acceptance-based treatment of tinnitus distress. Behaviour Research and Therapy,47(6), 523-528. doi: 10.1016/j.brat.2009.02.002 
Herzberg, K. N., Sheppard, S. C., Forsyth, J. P., Credé, M., Earleywine, M., \& Eifert, G. H. (2012). The Believability of Anxious Feelings and Thoughts Questionnaire (BAFT): A psychometric evaluation of cognitive fusion in a nonclinical and highly anxious community sample. Psychological Assessment, 24(4), 877-891. doi:10.1037/a0027782

Hinton, M. J., \& Gaynor, S. T. (2010). Cognitive defusion for psychological distress, dysphoria, and low self-esteem: A randomized technique evaluation trial of vocalizing strategies. International Journal of Behavioral Consultation and Therapy, 6(3), 164-185. doi: $\underline{10.1037 / \mathrm{h} 0100906}$

Hisli Şahin, N., \& Yeniçeri, Z. (2015). “Farkındalık” Üzerine Üç Araç: Psikolojik Farkındalık, Bütünleyici Kendilik Farkındalığı ve Toronto Bilgece Farkındalık Ölçekleri [Three Tools on "Awareness": Psychological Awareness, Integrative Self Awareness and Toronto Wisdom Awareness Scales]. Türk Psikoloji Dergisi, 30(76), 48-64.

Hooper, N., \& McHugh, L. (2013). Cognitive defusion versus thought distraction in the mitigation of learned helplessness. Psychological Record, 63, 209-218. doi:10.11133/j.tpr.2013.63.1.016

Hu, L., \& Bentler, P. M. (1999). Cutoff criteria for fit indexes in covariance structure analysis: Conventional criteria versus new alternatives. Structural Equation Modeling: A Multidisciplinary Journal, 6(1), 1-55. doi:10.1080/10705519909540118

Kline, R. B. (2011). Principles and practice of structural equation modeling (3rd ed.). New York: Guilford Press. Retrieved from http://scholar.google.com/scholar?hl=en\&btnG=Search\&q=intitle:Assumptions+in+St ructural+Equation+Modeling\#4

Larsson, A., Hooper, N., Osborne, L. A., Bennett, P., \& McHugh, L. (2016). Using brief cognitive restructuring and cognitive defusion techniques to cope with negative thoughts. Behavior Modification, 40(3), 452-482. doi: 10.1177/0145445515621488

Lau, M. A., Bishop, S. R., Segal, Z. V., Buis, T., Anderson, N. D., Carlson, L., ..., \& Devins, G. (2006). The Toronto mindfulness scale: Development and validation. Journal of Clinical Psychology, 62(12), 1445-1467. doi: $\underline{10.1002 / j c l p .20326}$

Luoma, J. B., Hayes, S. C., \& Walser, R. D. (2007). Learning ACT: An acceptance \& commitment therapy skills-training manual for therapists. New Harbinger Publications.

Mandavia, A., Masuda, A., Moore, M., Mendoza, H., Donati, M. R., \& Cohen, L. L. (2015). The application of a cognitive defusion technique to negative body image thoughts: A preliminary analogue investigation. Journal of Contextual Behavioral Science, 4(2), 8695. doi: 10.1016/j.jcbs.2015.02.003

Masuda, A., Twohig, M. P., Stormo, A. R., Feinstein, A. B., Chou, Y.-Y., \& Wendell, J. W. (2010). The effects of cognitive defusion and thought distraction on emotional discomfort and believability of negative self-referential thoughts. Journal of Behavior Therapy and Experimental Psychiatry, 41(1), 11-17. doi:10.1016/j.jbtep.2009.08.006

McCracken, L. M., Barker, E., \& Chilcot, J. (2014). Decentering, rumination, cognitive defusion, and psychological flexibility in people with chronic pain. Journal of Behavioral Medicine, 37(6), 1215-1225. doi:10.1007/s10865-014-9570-9 
Meunier, B., Atmaca, S., Ayranci, E., Gökdemir, B. P., Uyar, T., \& Baştuğ, G. (2014). Psychometric properties of the Turkish version of the Acceptance and Action Questionnaire-II (AAQ-II). Journal of Evidence-Based Psychotherapies, 14(2), 179-196.

Moffitt, R., Brinkworth, G., Noakes, M., \& Mohr, P. (2012). A comparison of cognitive restructuring and cognitive defusion as strategies for resisting a craved food. Psychology \& Health, 27(2), 74-90. doi:10.1080/08870446.2012.694436

Pilecki, B. C., \& Mckay, D. (2012). An experimental investigation of cognitive defusion. The Psychological Record, 62, 19-40. doi:10.1007/BF03395784

Roberts, S. L., \& Sedley, B. (2016). Acceptance and commitment therapy with older adults: Rationale and case study of an 89-year-old with depression and generalized anxiety disorder. Clinical Case Studies, 15(1), 53-67. doi:10.1177/1534650115589754

Schumacker, R. E., \& Lomax, R. G. (2010). A beginners guide to structural equation modeling. New York: Routledge.

Swank, J. M., \& Mullen, P. R. (2017). Evaluating evidence for conceptually related constructs using bivariate correlations. Measurement and Evaluation in Counseling and Development, 50(4), 270-274. doi: $10.1080 / 07481756.2017 .1339562$ 
The ethics committee approval for this study was obtained from the Ethics Committee of the Rectorate of Middle East Technical University, dated 13/04/2015 and numbered 28620816/177-361.

\section{Statement of Contribution of Researchers to the Article:}

1 st author contribution rate: $60 \%$

2nd author contribution rate: $40 \%$

Conflict of Interest Statement:

There is no conflict of interest.

Statement of Financial Support or Acknowledgment:

No financial support was received from any institution for this study. 